\title{
La voluntad de saber como instancia de la investigación foucaultiana sobre la biopolítica y la política
}

\author{
Marcelo Raffin ${ }^{1}$
}

\begin{abstract}
Resumen
Este artículo pretende realizar una hermenéutica de La voluntad de saber (1976) que permita comprender esta obra en su doble condición de constituir tanto el primer resultado de una investigación referida a una genealogía o historia de la sexualidad en el Occidente moderno, como el marco en el que Foucault desarrolla su análisis sobre la biopolítica y la gubernamentalidad y, sobre todo, en el que presenta las nociones de aquello que entiende por política. A tal fin, el artículo analiza, en primer lugar, la especificidad de La voluntad de saber como primer jalón de una genealogía de la sexualidad en el Occidente moderno. En segundo lugar, examina el emplazamiento de La voluntad de saber como inauguración oficial de los estudios sobre biopolítica y como punto de clivaje entre dos nociones fundamentales que, en opinión del autor, Foucault sostiene acerca de la política: una de carácter diagnóstico (ligada a la nociones de biopolítica y de gubernamentalidad) y otra de carácter propositivo (vinculada a los modos de subjetivación y las prácticas de sí). Finalmente, se subraya la relación de la subjetividad con los juegos de verdad, atravesada por el problema del poder-gobierno, como la piedra de toque de las preocupaciones y los propósitos generales de las investigaciones foucaultianas.
\end{abstract}

Palabras clave: Foucault. Biopolítica. Sexualidad.

The Will to Knowledge as an instance of the Foucauldian research work on biopolitics and politics

\begin{abstract}
This article pretends to realize a hermeneutics of The Will to Knowledge (1976) allowing to comprehend this work in its double condition: the first result of a research work concerning a genealogy or history of sexuality in the modern West and the framework in which Foucault develops his analysis on biopolitics and governmentality and in which he presents his notions of politics. To that end, the article analyzes, in the first place, the specificity of The Will to Knowledge as the first step of a genealogy of sexuality in the modern West. In the second place, it examines The Will to Knowledge as the official inauguration of the studies on biopolitics and as the turning point of two fundamental notions proposed, in the author's opinion, by Foucault about politics: one of a diagnostic character (linked to the notions of biopolitics and governementality) and another of a proposal character (related to the subjetivation modes and the practices of the self). Finally, the article underlines the relationship of the subjectivity with the games of truth marked by the problem of powergovernment as the touchstone of the preoccupations and general goals of the Foucauldian research works.
\end{abstract}

Keywords: Foucault. Biopolitics. Sexuality.

\footnotetext{
${ }^{1}$ HDR (Habilitation à diriger des recherches) en Filosofía y Doctor en Filosofía por la Universidad de París VIII, Investigador en Filosofía del CONICET en el Instituto de Investigaciones Gino Germani y Profesor Titular Regular de Filosofía en la Facultad de Ciencias Sociales, Universidad de Buenos Aires (UBA), Argentina. Ha publicado, entre otros libros, Droits de l'homme, sujet et devenir. L'expérience contemporaine du Cône sud d'Amérique latine (2017), La noción de política en Agamben, Esposito y Negri (editor, 2015) y Estética y política en la filosofía de Giorgio Agamben (editor, 2015). E-mail: raffinmarcelo@yahoo.com
} 


\section{Introducción}

El proyecto de "Historia de la sexualidad" es la investigación que Foucault dedica, en un primer momento, a la biopolítica para ser reformulado, clara y rápidamente, en la relación del sujeto con la verdad atravesada por el problema del poder entendido como gobierno, relación que, se podría sostener (como el propio Foucault lo hace), define el trabajo de toda su vida. En efecto, es Foucault mismo quien sienta las bases de esta relación ya en su primer curso en el Collège de France (1970-1971) que lleva por título (de manera coincidente con el del primer tomo de "Historia de la sexualidad") Lecciones sobre la voluntad de saber, donde recapitula sus trabajos previos y lanza sus investigaciones por venir, a los que nos propone caracterizar como "otros tantos fragmentos para una morfología de la voluntad de saber" (Foucault, 2011: 3$)^{2}$ y que finalmente elige, en 1983 y 1984, como los ejes centrales que permiten releer su "obra" 3 aunque redefinidos en términos de modos de veridicción, técnicas de gubernamentalidad y prácticas de sí (cf. Foucault, 2009: 10 y 2008: 3-8 y 42). Es por esta razón que, en mi opinión, "Historia de la sexualidad" en parte es una investigación sobre la sexualidad en Occidente y en parte no consiste solo en ello. En parte lo es en la medida en que Foucault pretende dar cuenta de la producción histórica de las categorías de sexualidad y de sexo en las sociedades burguesas occidentales en el siglo XIX. Y en parte no consiste solo en ello porque el proyecto no se detiene allí o no se resume solo en ello, sino que Foucault se interesa en la sexualidad en la medida en que es expresión de la biopolítica. Esta es definida por el filósofo como el acontecimiento decisivo de la modernidad, el que marca el

\footnotetext{
${ }^{2}$ Toda vez que no se indique lo contrario, la traducción es propia.

${ }^{3}$ Empleo el término "obra" siendo consciente de la crítica que Foucault formula a esta noción. De todas formas, y como lo señala Didier Éribon en la biografía que le dedica (Michel Foucault (1926-1984). Paris: Flammarion, 1989), como académico, Foucault entró en una trama de relaciones que lo hizo transformarse en el "autor" de una "obra" respecto de la que se desarrolló un aparato de "comentarios" que pretendieron y pretenden aún restituir su producción a esas figuras de las que siempre buscó desprenderse. Es entonces en este sentido que utilizo el término.
} 
tono y define la lógica moderna, como la relación entre el poder y la vida por la cual el poder da una cierta forma a la vida tratando de que no asuma otras, o como él mismo dice al presentar formalmente el concepto en La voluntad de saber (1976), tomo I de Historia de la sexualidad, como "aquello que hace entrar la vida y sus mecanismos en el ámbito de los cálculos explícitos y hace del poder-saber un agente de transformación de la vida humana” (Foucault, 1995: 188). La investigación de "Historia de la sexualidad" asumirá pues, a partir de La voluntad de saber y luego de ella, los contornos y el objetivo específico de analizar una tecnología política de la vida en los dos polos de la individualidad y de las poblaciones como tecnología del sexo y más allá de él.

En consecuencia, este artículo pretende realizar una hermenéutica de La voluntad de saber, que permita comprender esta obra en su doble condición de constituir tanto el primer resultado de una investigación referida a una genealogía o historia de la sexualidad en el Occidente moderno, como el marco en el que Foucault desarrolla su análisis sobre la biopolítica y la gubernamentalidad y, sobre todo, en el que presenta las nociones de aquello que entiende por política.

A continuación, me dedicaré entonces a analizar los siguientes puntos:

1. La especificidad de La voluntad de saber como introducción y primer resultado de una genealogía de la sexualidad en el Occidente moderno;

2. El emplazamiento de La voluntad de saber como inauguración oficial de la investigación sobre la biopolítica; 3. El problema político que subyace en La voluntad de saber y, más en general, en la investigación "Historia de la sexualidad", como punto de clivaje entre dos nociones fundamentales que, en mi opinión, Foucault sostiene acerca de la política: una de carácter diagnóstico, que queda resumida en la nociones de biopolítica y de 
gubernamentalidad, y otra de carácter propositivo, que desarrolla frente a ella a partir de los modos de subjetivación y las prácticas de sí, en la que las prácticas de veridicción cobran particular relevancia como prácticas de resistencia, creación y libertad; $y$

4. La relación de la subjetividad con la verdad o mejor, con los juegos de verdad, atravesada por el problema del podergobierno, como la piedra de toque de las preocupaciones y los propósitos generales de las investigaciones foucaultianas.

\section{La voluntad de saber como primer resultado de una genealogía de la sexualidad en el Occidente moderno}

Ciertamente, en un sentido fuerte, La voluntad de saber se presenta, específicamente, como la introducción y el primer jalón de una genealogía de la sexualidad en el Occidente moderno. En efecto, en esta primera obra que da inicio a la serie de trabajos que integran su investigación de "Historia de la sexualidad", Foucault se propone recorrer los discursos destinados a decir la verdad sobre el sexo (lo que denomina la "puesta en discurso" del sexo) pero, sobre todo, pretende analizar la "voluntad de saber" que los informa (a la vez como soporte e instrumento) y la intención estratégica que los sostiene como técnica de poder (cf. Foucault, ibíd.: 16). El propósito de Foucault es, por lo tanto, hacer la historia de las instancias de producción discursiva, de poder y de saber sobre el sexo y sus transformaciones, con el fin de demostrar que la "puesta en discurso" del sexo, lejos de haber sido sometida a un proceso de restricción (tal como sostiene lo que denomina la "hipótesis represiva") fue, por el contrario, el producto de un mecanismo de incitación y proliferación crecientes. Asimismo, con ello Foucault quiere demostrar que las técnicas de poder llevaron a la diseminación y a la implantación de sexualidades polimorfas y que la voluntad de saber sobre el sexo desembocó en una "ciencia de la sexualidad". Son estos movimientos los que constituyen el objetivo 
específico de la genealogía sobre el sexo y el dispositivo de sexualidad, que Foucault analizará a partir de ciertos hechos históricos que poseen el valor de marcas.

En este sentido, La voluntad de saber, como estudio específico sobre el dispositivo de sexualidad y la noción de sexo que este produce, retoma claramente la idea foucaultiana de la construcción histórica de una "voluntad de saber" (en clara ascendencia nietzscheana) ligada a la producción de regímenes de verdad, que el filósofo ya había planteado en su primer curso en el Collège de France. En consecuencia, su estudio sobre la producción de la sexualidad moderna como dominio de poder-saber, aparece como una propuesta de análisis que concibe la experiencia de la sexualidad como el resultado de sistemas de saber y de modalidades de poder (a mayor abundamiento sobre esta dimensión de análisis, cf. Davidson, 1987/2004: 30-65). Esta precisión de orden teórico anima toda La voluntad de saber y es presentada claramente al inicio, cuando Foucault cuestiona y pretende revertir la "hipótesis represiva” que caracterizaría las sociedades modernas occidentales, en particular, a partir de la implantación y la difusión de la moral victoriana del siglo XIX. Esta hipótesis no implica prohibir toda palabra sobre el sexo, sino su contario: incitar a la proliferación de los discursos sobre él, una de cuyas variables fundamentales es el secreto. Estos discursos sobre el sexo no solo refieren a la relación subjetiva con él (con la forma de desplegar y vivir la experiencia sexual) o institucional (por parte de las instituciones sociales o el Estado), sino que provienen de distintos lugares de saber y de producción de saber como la demografía, la biología, la medicina, la psiquiatría, la psicología, la moral, la pedagogía, la crítica política, la justicia penal y todos los controles sociales que proliferaron desde fines del siglo XIX.

El cuestionamiento propuesto por Foucault de la "hipótesis represiva” persigue varios objetivos: 
- reubicar esta hipótesis sobre la represión en una economía general de los discursos sobre el sexo al interior de la sociedades modernas desde el siglo XVII;

. analizar los efectos de poder inducidos por lo que se dice sobre la sexualidad;

- examinar los vínculos que se establecieron entre esos discursos, esos efectos de poder y los placeres que producen; $\mathrm{y}$ . auscultar el saber que se formaba a partir de ellos.

En una palabra, tal como lo resume Foucault, "se trata de determinar, en su funcionamiento y en sus razones de ser, el régimen de poder-saber-placer que sostiene en nosotros el discurso sobre la sexualidad humana" (Foucault, 1995: 19).

Pero es sobre todo en el punto $3^{\circ}$ "Dominio" (« Domaine ») de la IV parte del libro, donde Foucault analiza en particular la producción del campo de la sexualidad a través del dispositivo de sexualidad. En este análisis, la sexualidad aparece como punto de pasaje de las relaciones de poder que pueden servir de bisagra a las estrategias más variadas. En este sentido, el filósofo identifica cuatro grandes conjuntos estratégicos a partir del siglo XVIII: la histerización del cuerpo de la mujer, la pedagogización del sexo del niño, la socialización de las conductas procreadoras y la psiquiatrización del placer perverso, a los que hace corresponder, respectivamente, las figuras de la mujer histérica, el niño masturbador, la pareja malthusiana y el adulto perverso. De lo que se trata, en estas estrategias, es de la producción misma de la sexualidad como dispositivo histórico y como dispositivo político.

Foucault señala que el dispositivo de sexualidad desarrollará un juego particular con relación al dispositivo de alianza, otro gran dispositivo que ha perdurado en las sociedades occidentales. En este juego se inscribe el papel de la familia, que actuará como un cristal respecto del dispositivo de sexualidad en la medida en que parece difundir una sexualidad que, en realidad, refleja y difracta (cf. Foucault, ibíd.: 147). Según Foucault, en este espacio de juego, vino 
a alojarse el psicoanálisis pero modificando considerablemente el régimen de las inquietudes y los reaseguros. El filósofo explica que es claro que al principio el psicoanálisis pudiese suscitar desconfianza y hostilidad puesto que,

llevando al extremo la lección de Charcot [de separar al "enfermo" de su familia con el fin de que, para observarlo mejor, se la escuchara lo menos posible], se proponía recorrer fuera del control familiar, la sexualidad de los individuos; sacaba a la luz esa sexualidad misma sin recubrirla con el modelo neurológico; más aún, ponía en cuestión las relaciones familiares con el análisis que hacía de ellas (cf. Foucault, ibíd.: 148).

Sin embargo, pese a que el psicoanálisis, a través de sus modalidades técnicas, parecía ubicar la confesión de la sexualidad fuera de la jurisdicción familiar, reencuentra en el corazón mismo de esa sexualidad, como principio de su formación y cifra de su inteligibilidad, la ley de la alianza. La garantía de que en el fondo de la sexualidad de cada quien, se encuentra la relación padres-hijos, permite mantener la unión del dispositivo de sexualidad y el dispositivo de alianza.

Es necesario recordar que estos análisis de Foucault se basan en una fuerte crítica a lo que denomina la "representación jurídica" del poder que informa el psicoanálisis (al menos en sus formulaciones “clásicas”), básicamente en lo que refiere a la forma de comprender el deseo sobre la base de la ley. Foucault explicita la crítica a este modo de concebir el poder, que no solo atañe al psicoanálisis, sino a la teoría política en general, en términos jurídico-discursivos, es decir, como poder-ley, como poder soberano cuya forma general es el derecho. A él opone, a los fines analíticos (y no de manera prescriptiva), la necesidad de una noción de poder "normalizador" (pese a que en La voluntad de saber no utiliza este término que corresponde más bien a la manera en que conceptualiza el poder en Los anormales, el curso que precede a la publicación del libro) como "tecnología" y control sobre la vida y los cuerpos 
vivientes. Foucault insiste, en consecuencia, en la necesidad de emplear una noción de "analítica" del poder en términos productivos, estratégicos y positivos ("modelo estratégico"), en la medida en que si bien muchas de las formas de la "representación jurídica” del poder subsistieron y subsisten aún, nuevos mecanismos de poder que tomaron a su cargo la vida de los hombres y que son, por lo tanto, irreductibles a ese tipo de representación, lo han ido penetrando paulatinamente. Son estos mecanismos los que responden antes bien al diagrama que configura la biopolítica.

Ahora bien, hacia el final de La voluntad de saber, Foucault plantea una distinción fundamental entre sexualidad y sexo que se puede aplicar a otros objetos y casos históricos que estudia (como, por ejemplo, la locura, la enfermedad o la delincuencia): no solo la sexualidad es producida como tal a partir del juego que se establece entre discursos, prácticas y tecnologías de poder y de saber (informadas por una cierta "voluntad de saber"), sino que también el sexo como tal, es producido al interior de ese juego. En este sentido, no hay una producción históricamente connotada de la sexualidad (como correlato del tratamiento del sexo en la modernidad occidental) y, al margen de ello, como un objeto transhistórico o ahistórico, el sexo, sino que, en la perspectiva foucaultiana, este objeto, esta "realidad", también es producido al interior del juego entre poder, saber, verdad y formas de la subjetividad que caracteriza una determinada formación sociohistórica como la moderna. Así, es el dispositivo de sexualidad el que hace aparecer la noción de sexo como elemento especulativo y necesario para su funcionamiento pero invirtiendo el principio de su proceso de producción. La noción de sexo así producida por el dispositivo de sexualidad, hace aparecer a la sexualidad como dependiente del sexo, ubicándolo del lado de lo real y de lo natural y emplazándose a sí misma en el ámbito de lo artificial. En este sentido, Foucault afirma enfáticamente que es necesario "no referir a la instancia del sexo una historia de la sexualidad, sino mostrar 
cómo 'el sexo' se encuentra bajo la dependencia histórica de la sexualidad" (Foucault, ibíd.: 207).

En esta operación reside el origen, sostiene Foucault, de una "teoría general del sexo" que ejerció sobre el dispositivo de sexualidad un número de funciones que la volvieron indispensable, instituyendo una unidad y una contigüidad artificiales entre lo biológico, lo anatómico, lo afectivo y lo identitario, y las formas de una sexualidad normal y una sexualidad patológica.

En consecuencia, el sexo no es más que un punto ideal que se ha vuelto necesario por el dispositivo de sexualidad y por su funcionamiento. Es por ello que Foucault sostiene que antes que de la sexualidad, de lo que debemos liberarnos es del sexo y del deseo (del deseo del sexo) como uno de sus principios de funcionamiento más esenciales. Por esta razón, concluye, contra el dispositivo de sexualidad, el punto de apoyo del contraataque no debe ser el sexodeseo, sino los cuerpos y los placeres.

El proyecto de "Historia de la sexualidad" como genealogía del dispositivo de sexualidad y del sexo, se completa en los tomos II y III (L'usage des plaisirs y Le souci de soi), con el análisis de la constitución del hombre occidental como sujeto de deseo a través de la elaboración de una "hermenéutica del sujeto", donde el problema de la verdad, la verdad, a través de un cierto número de modos de veridicción, desempeñará un papel fundamental. Entre esos modos de veridicción, la confesión (l’aveu) y las prácticas de sí se presentarán como fundamentales para la relación que el sujeto mantiene consigo mismo tanto en términos de un poder que sujeta y domina como también en términos de ejercicio de poder resistente y creador. Vale decir que esa hermenéutica del sujeto y las prácticas de sí tendrán la duplicidad de poder actuar tanto en uno como en otro sentido. 


\section{La voluntad de saber como instancia de definición de la investigación sobre la biopolítica}

Es fundamental restituir la investigación que Foucault propone bajo el título de "Historia de la sexualidad", y dentro de ella La voluntad de saber, al contexto de producción de la biopolítica, que el filósofo explicitará como "gubernamentalidad" en una doble dimensión de poder sobre la vida individual (el sujeto, la subjetividad individual, su "cuerpo" y su "alma") y la vida colectiva (las poblaciones). En este sentido, Foucault mismo sostiene que el sexo constituye verdaderamente una cuestión política, un "desafío" o un "reto" político ( enjeu politique »), en la medida en que aparece articulando los dos ejes a lo largo de los cuales se desarrolló toda la tecnología política de la vida en la modernidad: el de la disciplina y el de la regulación de las poblaciones (cf. Foucault, ibíd.: 191). Foucault refuerza esta idea señalando explícitamente que "el sexo es acceso a la vez a la vida del cuerpo y a la vida de la especie" (Foucault, ibíd.: 192).

El dispositivo de sexualidad, pieza clave de la "historia de la sexualidad" en el siglo XIX occidental, no es, sin embargo, el único acontecimiento fundamental de la biopolítica para Foucault. Al presentarlo, el filósofo señala, al mismo tiempo, el de la "simbólica de la sangre", con el que el primero va a establecer una relación muy especial puesto que, bajo la matriz biopolítica, el dispositivo de sexualidad será siempre preeminente pero respecto del cual, la "simbólica de la sangre" (que había jugado un papel decisivo en los siglos previos al XIX) se transformará en racismo moderno.

En este sentido, no se podría dejar de vincular los desarrollos sobre la biopolítica, el dispositivo de sexualidad y el racismo moderno con la reformulación que Foucault plantea acerca de su diagnóstico fundamental de la modernidad: de la sociedad de normalización a los dispositivos de seguridad y la noción de gubernamentalidad. Así, "Historia de la sexualidad" se vincula tanto con los desarrollos que Foucault presenta en Vigilar y castigar y los 
cursos y conferencias a él asociados (los que dicta desde 1972 a 1975 -Teorías e instituciones penales, La sociedad punitiva, El poder psiquiátrico y Los anormales- y las conferencias de Río de Janeiro de 1973 y 1974) y el curso que acompaña la publicación de La voluntad de saber (Defender la sociedad) y su colofón de 1978 y 1979 (Seguridad, territorio, población y Nacimiento de la biopolítica). El curso de 1978 está dedicado especialmente a la cuestión de la biopolítica y sienta las bases de la reformulación del problema del poder hacia el problema del gobierno y de su vinculación con el de la verdad mediante la noción de gubernamentalidad.

Asimismo, si tenemos en cuenta el proyecto inicial de "Historia de la sexualidad", aquel que Foucault presenta en la contratapa de la publicación original de La voluntad de saber, en 1976, que incluía seis tomos (La volonté de savoir, La chair et le corps, La croisade des enfants, La femme, la mère et l'hystérique, Les pervers y Populations et races), y la deriva definitiva que el proyecto asumió, podremos constatar que el proyecto originario fue llevado a cabo pese a que luego del primer tomo, solo se sucedieron tres más (L'usage des plaisirs, Le souci de soi y Les aveux de la chair). En efecto, se puede afirmar que algunos de los cursos que Foucault dictó durante los años de trabajo sobre el proyecto de "Historia de la sexualidad", desarrollaron efectivamente los objetos de los tomos que restaban al tiempo que acompañaron la reformulación del proyecto original.

\section{La voluntad de saber como el inicio del clivaje entre las dos nociones fundamentales de la política en Foucault}

En mi opinión, además de la especificidad de constituir una genealogía de la sexualidad en Occidente y el marco general de la investigación sobre la biopolítica, el proyecto de "Historia de la sexualidad" articula dos conceptos fundamentales con los que Foucault concibe la política. Por un lado, el que aparece ligado a la nociones de biopolítica y gubernamentalidad, como momento 
culminante de su análisis de la producción de la vida en su relación con el gobierno/poder en la modernidad, y por el otro, el que refiere al ejercicio de la resistencia en términos de modos de subjetivación y prácticas de sí como espacio de libertad y creación. La voluntad de saber se encuentra en el umbral de la torsión que Foucault hará jugar en los años inmediatos posteriores entre ambos conceptos de la política, que reconoce su punto de clivaje en el objetivo de desentrañar de qué manera es producida la sociedad moderna en la tensión entre prácticas de veridicción (economía) y prácticas de jurisdicción (derecho), como mecanismos interno y externo, respectivamente, de configuración de la vida en su relación con el gobierno.

El primer concepto de la política en Foucault está ligado a las nociones de biopolítica y gubernamentalidad. Estas refieren a una relación muy particular entre la vida y el gobierno/poder por la cual la primera es modelada por el segundo, apresada, sujetada, sometida por él y a él, y, sobre todo, configurada de una cierta forma que impide cualquier otra posibilidad. En particular, ambas nociones pretenden dar cuenta de un fenómeno específico de la sociedad moderna como es la configuración de la vida (cuyo epifenómeno central es la ecuación entre animalidad y politicidad), en el marco más general de la propuesta de análisis foucaultiano de la producción de la sociedad moderna como correlato del mercado. La noción de la política ligada a la biopolítica y a la gubernamentalidad tiene más bien un valor diagnóstico o analítico del estado de cosas vigente.

Ahora bien, hacia fines de la década de 1970, la investigación de "Historia de la sexualidad" dará un giro fundamental puesto que, de aquí en adelante, se centrará en los modos en que la subjetividad se relaciona consigo misma por referencia al gobierno y a la verdad. La relación había sido ya planteada, sin embargo, por Foucault en el curso de 1978, en el que sienta las bases de la biopolítica y la gubernamentalidad y vincula claramente ambas nociones a la verdad en la tradición de la pastoral cristiana. El estudio del 
pastorado cristiano permite a Foucault remontarse a la Antigüedad, donde va a encontrar un modelo diferente para pensar esa relación como base de otro sentido de la política. Asimismo, cabe señalar que la posibilidad de la resistencia al poder, a su vez, como ejercicio de poder, ya había sido formulada por Foucault en los cursos "biopolíticos" del Collège de France (1976-1979).

De esta suerte, Foucault propondrá una noción de la política diferente o en sentido inverso a la que queda definida por la biopolítica y la gubernamentalidad. Esta nueva noción, elaborada, de todas formas, por relación a esas categorías, consiste en la posibilidad de resistir a ellas y, eventualmente, crear o instituir un nuevo orden. Este otro concepto de la política se vincula con lo que tradicionalmente se refiere como la dimensión "poiética" de la política y posee más bien un carácter propositivo.

Esta noción de política en Foucault como resistencia a la gubernamentalidad, es desarrollada por el filósofo a partir de los conceptos de modos de subjetivación y de prácticas de sí, inscriptos en el marco más general de las formas de producción o manifestación de la verdad o "aleturgía”. De esta manera, en el pasaje de los años 1970 a los años 1980, esta noción de la política tomará como punto de apoyo los modos en que el sujeto se relaciona consigo mismo por referencia al gobierno y a la verdad o, como Foucault dice en este momento, reformulando y afinando los términos, por referencia a las "técnicas de gubernamentalidad" y a los "modos de veridicción”. Así, Foucault piensa diferentes "prácticas de sí" que rastreará en el mundo antiguo pero para pensar el mundo moderno.

Las prácticas de sí reconocen como base conceptual la “inquietud" o "preocupación por sí mismo" (souci de soi), como trabajo sobre sí que implica también y al mismo tiempo, a los otros o, para decirlo de otra manera, que está atravesado por la dimensión de la alteridad. Preocuparse por uno mismo es, en la interpretación foucaultiana, preocuparse también por los otros, no solo en la medida en que esta preocupación va a estar dirigida también a los 
otros, sino además porque, aun concebida desde la instancia meramente individual, implica a los otros.

Es necesario destacar, además, que la noción de "preocupación por sí mismo" en Foucault, remite al gobierno y a la política. El « souci de soi » expresa un proyecto político que pasa también y al mismo tiempo, por un proyecto ético en la medida en que al elaborar esta noción, Foucault está interesado en desarrollar las formas o los modos de subjetivación, es decir, las formas o modos en que el sujeto se relaciona consigo mismo por referencia al gobierno y a la verdad. El espacio que queda entre ellos es el de la libertad.

Ahora bien, el "souci de soi » aparece enmarcado muy particularmente en la investigación foucaultiana, en una práctica de subjetivación específica, como es la "parresía", es decir, el decir verdadero, auténtico o franco, que Foucault presenta hacia el final de La hemenéutica del sujeto (curso del Collège de France de 19811982 específicamente dedicado a la inquietud de sí) y desarrolla en sus dos últimos cursos en esa institución (en 1983 y 1984). La parresía, como modo de relación del sujeto consigo mismo por referencia al gobierno y a la verdad, remite a un sujeto resistente, a la idea de la existencia como "obra" y como obra bella y a la relación entre el vivir de un cierto modo y el abocarse a decir la verdad.

Es en este punto donde, creo, aparece la clave que permite pensar el « souci de soi » a través de la parresía como práctica de sí, como un instrumento de construcción ético-política. La parresía permite hacer coincidir el pensamiento con la acción pero solo en la medida en que es acción sobre sí junto con otros. El dar forma y estilo a una vida permite romper los moldes que hacen que la vida y su mundo sean solo concebidos en un solo sentido, es decir, permite pensar otra vida y otro mundo (autre vie/autre monde) como una vida y un mundo diferentes (vie autre/monde autre) al que vivimos y como espacio de libertad. Este trabajo de imaginación y de construcción de vidas y mundos diferentes solo es posible junto con otros. De esta manera, el "souci de soi » es también, al mismo 
tiempo, " souci des autres », porque la parresía solo pueda tener lugar junto con otros.

\section{Epílogo}

Llegados a este punto, podemos preguntarnos cuál es el significado de La voluntad de saber, tomo I de Historia de la sexualidad, como estudio específico y como pieza de investigación en la producción foucaultiana considerada en su conjunto. Sin dejar de reconocer la especificidad de la contribución que el libro significa respecto del desarrollo de una genealogía de la sexualidad en Occidente, creo que es necesario subrayar la tensión en la que se inscribe en la investigación foucaultiana sobre la producción moderna de la subjetividad, la verdad y el gobierno en el doble sentido de la dominación y la resistencia. Por ello, considero que La voluntad de saber constituye, además, el momento inicial de un plan de trabajo en el que confluyen, a partir de una torsión, dos nociones fundamentales sobre la política en Foucault que definen fuertemente la producción global de ideas del filósofo, una de carácter diagnóstico ligada a la biopolítica y la gubernamentalidad y otra de carácter propositivo vinculada a los modos de subjetivación y las prácticas de sí. De allí que, en mi opinión, el proyecto de "Historia de la sexualidad", en el que se inscribe La voluntad de saber como primer resultado parcial, debe ser entendido en la tensión entre el dispositivo de sexualidad y el problema de la relación entre el gobierno, los modos de veridicción y las formas de la subjetividad, como paradigma de las preocupaciones y los propósitos generales de las investigaciones foucaultianas.

\section{Referencia}

Davidson, A. (1987). "Sex and the Emergence of Sexuality", en Davidson, A. (2004) The Emergence of Sexuality. Historical Epistemology and the Formation of Concepts. Cambridge/London, Harvard University Press. 
Raffin, M. - | 239

Foucault, M. (2011). Leçons sur la volonté de savoir, Cours au Collège de France, 1970-1971. Paris, Gallimard/Seuil.

(1995). La volonté de savoir, tomo I de Histoire de la sexualité. Paris, Gallimard.

(2004). Sécurité, territoire, population, Cours au Collège de France, 19771978. Paris, Gallimard/Seuil.

(2001). L'herméneutique du sujet, Cours au Collège de France, 1981-1982, Paris, Gallimard/Seuil.

(2008). Le gouvernement de soi et des autres, Cours au Collège de France, 1982-1983. Paris, Gallimard/Seuil.

(2009). Le courage de la vérité. Le gouvernement de soi et des autres II, Cours au Collège de France. 1984. Paris, Gallimard/Seuil. 